\title{
Bubble interactions in liquid/gas flows
}

\author{
L. VAN WIJNGAARDEN
}

Technological University Twente, Enschede, The Netherlands

\begin{abstract}
The system of equations, usually employed for unsteady liquid/gas flows, has complex characteristics. This as well as other facts have led to the search for a more accurate description of effects associated with relative motion. For liquid/bubble systems the fluctuations resulting from hydrodynamic interaction between the bubbles may be taken into account in the same way as particle interactions in the theory of viscous suspensions. This is illustrated for the pressure. In a description accurate up till the third power of the void fraction two-bubble interactions are of primary importance. Numerically obtained results for the relative motion in bubble pairs are presented and interpreted with help of simplified equations from which conclusions can be drawn in an analy tic way.
\end{abstract}

\section{Introduction}

In recent years the numerical instabilities which arise in the computation of transients in two-phase flows, have stimulated research on the interaction between phases. Such interactions, inertial or of other nature, give rise to additional terms in the equations of motion. These terms might, in rendering real characteristics, prevent instabilities.

In our laboratory a study is in progress regarding the interaction effects in a mixture of massless spheres and a perfect liquid. Such a fluid reasonably approximates a bubbly flow under circumstances in which bubbles are small enough to be kept spherical by surface tension and in which surface active agents are absent in the liquid. The latter condition means that the flow around an individual bubble in the mixture can be with good accuracy approximated by a potential flow. The interaction effects produce in any point in the fluid fluctuating pressures and velocities. Just as in turbulence one is interested in mean values.

When a distinction between fluctuations and mean values is made and some type of averaging is carried out (ensemble averaging, volume averaging or otherwise) effects of the fluctuations on the stress in the fluid remain. These have been calculated by Voinov and Petrov [7] with the use of a cell method. Such a method however leads to results of unknown accuracy. Our approach is similar to that used by Batchelor and his associates, see e.g. [2] in the theory of suspensions dominated by viscous effects. As an illustration we deal here with the calculation of the average or bulk pressure in the inhomogeneous liquid. 


\section{Bulk pressure in bubbly liquid}

Consider a large volume $V$ of a suspension of $N$ bubbles of zero mass in a perfect liquid. A point in the fluid, whether in a bubble or in liquid, is indicated with its position vector $\mathbf{x}$. We assume that the ensemble average of the velocity $\mathbf{u}$ over all possible configurations of the $N$ bubbles is given as $\mathbf{U}_{0}$,

$$
\frac{1}{N !} \int \mathbf{u}\left(\mathbf{x}, C_{N}\right) P\left(C_{N}\right) \mathrm{d} C_{N}=\mathbf{u}_{0}
$$

where $P\left(C_{N}\right)$ is the probability distribution of $N$ bubbles, or

$$
\langle\mathbf{u}\rangle(\mathbf{x})=\mathbf{U}_{0} \text {. }
$$

The volume flow $\mathrm{U}_{0}$ can be divided in a gas flow (assuming that the bubbles are filled with gas of negligible density) $\alpha \mathbf{U}_{g}, \alpha$ being the concentration of gas by volume, and a volume flow $(1-\alpha) \mathbf{U}_{1}$ of liquid,

$$
\alpha \mathbf{U}_{g}+(1-\alpha)(1-\alpha) \mathbf{U}_{l}=\mathbf{U}_{0} .
$$

Our aim eventually is to formulate equations of motion for the averaged quantities. If we carry out the ensemble averaging, the average pressure $\langle p\rangle$ makes its appearance. Assuming that it is permitted to replace ensemble averaging by volume averaging (for this statistical homogeneity is required) we have

$$
\langle p\rangle=\frac{1}{V} \int_{V} p \mathrm{~d} V=\frac{1}{V} \int_{V_{l}} p \mathrm{~d} V+\sum \frac{1}{V} \int_{V_{B}} p \mathrm{~d} V .
$$

In this equation, $V_{l}$ denotes the volume occupied by liquid and $V_{B}$ the volume of one of the $N$ identical bubbles. Next we define $\langle p\rangle_{l}$ as the pressure averaged over the liquid alone. Then

$$
\begin{aligned}
\langle p\rangle & =(1-\alpha)\langle p\rangle_{l}+\sum \frac{1}{V} \int_{V_{B}} p \mathrm{~d} V \\
& =\langle p\rangle_{l}+\sum \frac{1}{V} \int_{V_{B}}\left\{p-\langle p\rangle_{l}\right\} \mathrm{d} V .
\end{aligned}
$$

Upon introducing the number density $n=N / V$ and the quantity $S$ given by

$$
S=\int_{V_{B}}\left(p-\langle p\rangle_{l}\right) \mathrm{d} V
$$

we write (4) as

$$
\langle p\rangle=\langle p\rangle_{l}+n\langle S\rangle .
$$

The quantity $S$ in (5) is similar to the 'particle stress' discussed in [1]. In particular it was shown there that (5) may be written as 


$$
S=\frac{1}{3} \int_{A_{B}}\left(p-\langle p\rangle_{l}\right) \mathbf{r} \cdot \mathrm{d} \mathbf{A},
$$

the integration being over a surface $A_{B}$ which lies just at the liquid side of the interface between liquid and gas.

When $\alpha \ll 1$ we may in a first approximation assume each bubble to be alone in the liquid. Far from the bubble, the pressure is $\langle p\rangle$ and the velocity $\mathbf{U}_{0}$. With bubble velocity $\mathbf{U}_{g}$, the flow is presented by the potential

$$
\phi=\mathbf{U}_{0} \cdot \mathbf{r}+\frac{\left(\mathbf{U}_{0}-\mathbf{U}_{\mathrm{g}}\right) \cdot a^{3} \mathbf{r}}{2 r^{3}},
$$

where $a$ is the radius of a bubble and $\mathbf{r}$ gives the position of a point with respect to the centre of the bubble. Upon calculation of $p$ with Bernoulli's Theorem ( $\mathbf{U}_{0}$ may depend on time) and upon carrying out of the integration in (7), we find for $S$,

$$
S \approx S_{0}=-\frac{\pi a^{3}}{3}\left\{\left|\mathbf{U}_{0}-\mathbf{U}_{g}\right|\right\}^{2}
$$

Accordingly we find for the bulk pressure, from (6),

$$
\langle p\rangle=\langle p\rangle_{l}-\frac{1}{4} \alpha \rho\left\{\left|\mathbf{U}_{l}-\mathbf{U}_{g}\right|\right\}^{2}+O\left(\alpha^{2}\right),
$$

$\rho$ being the density of the liquid. (Voinov and Petrov [7] find $\frac{1}{3}$ where $\frac{1}{4}$ occurs in [10].) After a similar calculation of the 'Reynolds stress', by calculation of $\langle\rho \mathbf{u u}\rangle$ we obtain, see $[10]$, to $O(\alpha)^{2}$, for one-dimensional flow in $x$ direction

$$
\rho(1-\alpha)\left\{\frac{\partial U_{l}}{\partial t}+U_{l} \frac{\partial U_{l}}{\partial x}\right\}=-\frac{\partial}{\partial x}\langle p\rangle_{l}+\frac{1}{20} \frac{\partial}{\partial x}\left\{\alpha\left(U_{g}-U_{l}\right)^{2}\right\}
$$

which may be compared with the result found by Van Beek [8] in his contribution to this Symposium.

In the next order of approximation, we start with the exact expression for $\langle S\rangle$,

$$
\langle S\rangle=\frac{1}{N !} \int S\left(\mathbf{x}_{0}, C_{N}\right) P\left(C_{N} / \mathbf{x}_{0}\right) \mathrm{d} C_{N},
$$

where $S$ is given by (5) for a bubble with centre in $\mathbf{x}_{0}$, and where $P\left(C_{N} / \mathbf{x}_{0}\right)$ is the probability distribution for $N$ bubbles, an additional one being in $\mathbf{x}_{0}$ (the so-called conditional probability). We now replace in each configuration the $N$ bubbles by just one bubble situated in $\mathbf{x}_{1}$ and with $P\left(\mathbf{x}_{1} / \mathbf{x}_{0}\right)$ as the probability of finding a bubble centred at $x_{1}$, when there is one centred in $\mathbf{x}_{\mathbf{0}}$, we have 


$$
\langle S\rangle=S_{0}+\int\left(S-S_{0}\right)\left(\mathbf{x}_{0}, \mathbf{x}_{1}\right) P\left(\mathbf{x}_{1} / \mathbf{x}_{0}\right) \mathrm{d}^{3} \mathbf{x}_{1}
$$

The integration in (13) is over all possible positions of the second bubble.

In $S$, as given by (5) or (7), this time $p$ is the pressure at distance $\mathbf{r}$ (see Figure 1) from the centre of one bubble, another one having its centre in $\mathbf{x}_{1}$. The potential for the flow involving two spheres can in the present context conveniently be expressed in terms of twin spherical expansions as used in [4] and [9]. When this is done and the associated pressure is introduced in (13) we are confronted with the fact that $\left(S-S_{0}\right)$ behaves at large values of $\left(\mathbf{x}_{1}-\mathbf{x}_{0}\right)$ like $\left\{\left|\mathbf{x}_{1}-\mathbf{x}_{0}\right|\right\}^{-3}$ and that therefore the integral is not uniformly convergent. This problem can be solved by using the normalization technique, reviewed in [2].

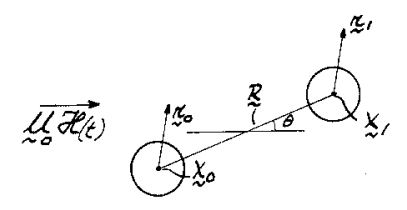

Figure 1. Two bubbles, in $x_{0}$ and $x_{1}$, immersed in a liquid which acquires a velocity $\mathrm{U}_{0} H(t)$.

Another problem is the determination of $P\left(\mathrm{x}_{1} / \mathrm{x}_{0}\right)$. In contrast to inhomogeneous media with a fixed structure, like a porous bed, the probability distribution is in general affected by the flow itself. The question whether a stationary distribution is reached can only be considered after the problem of determining relative motion in a pair of bubbles has been solved. Both for the calculation of $\left(S-S_{0}\right)$ and for the investigation of $P\left(\mathbf{x}_{1} / \mathbf{x}_{0}\right)$ we turn our attention to relative motion between two bubbles.

\section{Relative motion in a pair of bubbles}

To be specific we consider pairs of bubbles in a bubbly flow which is at $t=0$ instantaneously accelerated to a velocity $\mathbf{U}_{0}$. The equation of motion is for each bubble

$$
\int_{A_{0}} p \mathrm{~d} \mathbf{A}=0
$$

because the bubble mass is neglected. The pressure $p$ can be derived, by using Bernoulli's Theorem, from the potential of the flow. This consists, if we consider a specific bubble with centre in $\mathbf{x}_{0}$, of a part which is regular in $\mathbf{x}_{0}$, with gradient $\mathbf{u}_{R}$ and a singular part. The latter can be represented by monopoles, dipoles and multipoles situated in points $\mathbf{x}_{s}$ within the bubble (for a spherical bubble $\mathbf{x}_{s}$ coincides with $\mathbf{x}_{0}$ ). The force on a bubble can be expressed in the strength $\mathbf{M}_{q}$ of these singularities and the derivatives in $\mathbf{x}_{0}$ of $\mathbf{u}_{R}$. 
This has been done recently by Landweber and Miloh [6]. Here we disregard, for simplicity, changes of the volume $\tau$ of a bubble - inclusion offers no essentially new problems - and write using the result in [6] and denoting the velocity of a bubble with $\mathbf{v}$, the relation (14) as

$$
\frac{\mathrm{d}}{\mathrm{d} t}\left\{\rho \tau \mathbf{v}-4 \pi \mathbf{M}_{1}\right\}+\mathbf{F}=0
$$

with

$$
\mathbf{F}=-4 \pi \rho \sum \mathbf{M}_{q} \frac{\partial^{q}}{\partial \mathrm{x}^{q}}\left(\mathbf{u}_{R}\right)_{\mathrm{x}=\mathrm{x}_{\mathrm{s}}} .
$$

The part of the force which is indicated with $\mathbf{F}$ is due to the 'velocity squared' term in Bernoulli's Theorem. $\mathbf{M}_{q}$ is the singularity of order $q\left(\mathbf{M}_{1}\right.$ is a dipole, $\mathbf{M}_{2}$ a quadrupole etc.) and is multiplied in the expression for the force with the gradient of order $q$ of $\mathfrak{u}_{R}$ in $\mathrm{x}_{0}$. At time $t=0^{+}$only the terms in the braces in (15) are effective and the resulting velocity is, see [9], the same for each bubble and of magnitude $\mathbf{v}=3 \mathbf{U}_{0}+\mathrm{O}(\alpha)$. The $\mathbf{M}_{q}$ can be found from the potential mentioned above and equations for the velocities of the bubbles in $x_{0}$ and in $x_{1}$ can be found by applying (15) to each of them. These are complicated expressions because the $\mathbf{M}_{q}$ contain the unknown velocities of the bubbles. Note that because of the occurrence of $F$ relative motion with velocity

$$
\mathbf{V}=\frac{\mathrm{d} \mathbf{R}}{\mathrm{d} t}
$$

developes for $t>0, \mathbf{R}$ being the distance $\mathbf{x}_{1}-\mathbf{x}_{0}$ between the two centres. Next by combination of these equations an equation for $\mathbf{V}$ can be constructed, which has to be solved numerically. This program has been carried out in [5] by Knibbe. Some trajectories $\mathbf{R}=\mathbf{R}(t)$ obtained from this by Biesheuvel are shown in Figure 2.

Since the analysis is quite complicated, it is difficult to understand and interprete the results on the basis of the full problem. A qualitative insight can be obtained by taking only the leading singularities, in terms of the parameter $(a / R)$ into account. The leading term in $\partial / \partial x\left(\mathbf{u}_{R}\right)$ in $(16)$ is the gradient of the velocity induced in $x_{0}$ by the dipole in $x_{1}$. The latter behaves as $\mathrm{U}_{0} a^{3} / R^{3}$ and because the initial dipole strength is $\mathrm{U}_{0} a^{3}$ we can neglect the variation in the dipole strength due to the relative motion. The leading term therefore in $\mathbf{F}$ is

$$
\mathbf{F}_{0}=-4 \pi \rho a^{3}\left(\mathbf{U}_{0} \cdot \nabla_{0}\right) \mathbf{u}_{R}(\mathrm{o}),
$$

where $\mathbf{u}(0)$ indicates the velocity in $\mathbf{x}_{0}$ and $\nabla_{0}$ indicates the gradient with respect to $\mathbf{x}_{0}$. The velocity $\mathbf{u}_{R}(\mathrm{o})$ is given by 

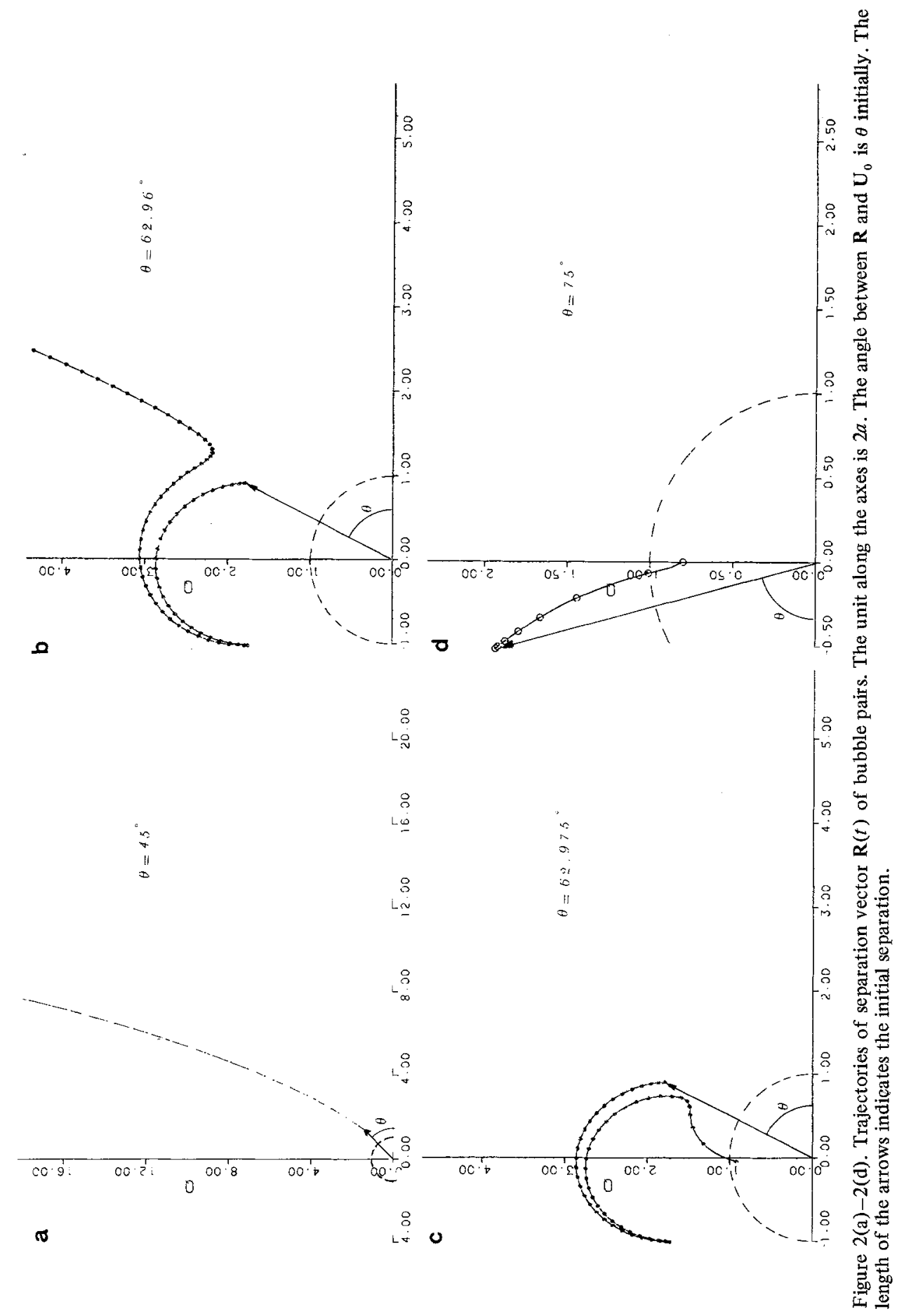

$\stackrel{\mathscr{g}}{\mathrm{F}}$ 


$$
\mathbf{u}_{R}(\mathrm{o})=-\nabla \frac{\mathbf{U}_{0} a^{3} \cdot \mathbf{r}_{1}}{r_{1}^{3}}
$$

and is in this approximation equal to $\mathbf{u}_{R}(1)$, the velocity induced in $\mathbf{x}_{1}$. From subtraction of the equations for $v_{0}$ and $v_{1}$, we find in this approximation

$$
\frac{\mathrm{d}}{\mathrm{d} t}\left\{-\frac{1}{2} \rho \tau\left(\mathbf{v}_{0}-\mathbf{v}_{1}\right)\right\}=8 \pi \rho a^{3}\left(\mathbf{U}_{0} \cdot \nabla_{R}\right) \nabla_{R} \frac{\mathbf{U}_{0} a^{3} \cdot \mathbf{R}}{R^{3}}
$$

With $\mathbf{v}_{1}-\mathbf{v}_{0}=\mathrm{d} \mathbf{R} / \mathrm{d} t$ and working out the right-hand side of $(20)$ we can write this as

$$
\frac{\mathrm{d} \dot{\mathbf{R}}}{\mathrm{d} t}+\nabla G=0
$$

with

$$
G=\frac{12 a^{3} U_{0}^{2}}{R^{3}}\left(3 \cos ^{2} \theta-1\right),
$$

$\theta$ being the angle between $\mathbf{U}_{0}$ and $\mathbf{R}$ as indicated in Figure 1 and the dot on $R$ indicating the time derivative. The relation (21) means that there is a constant of the motion, the energy, which is in spherical polar coordinates

$$
\frac{1}{2}\left(\dot{R}^{2}+R^{2} \dot{\theta}^{2}\right)+G=G_{0}, \text { say }
$$

where account has been taken of the initial conditions on $\dot{R}$ and $\dot{\theta}$.

From analytical mechanics it follows that there is a Lagrangian $L$,

$$
L=\frac{1}{2}\left(\dot{R}^{2}+R^{2} \dot{\theta}^{2}\right)-G,
$$

the Euler equations of which provide the equations of motion (20). These equations cannot be solved analytically but some important conclusions can nevertheless be drawn. Lack of space prevents to give the analysis here for which we refer to [3]. Here we summarize these conclusions, referring to Figure 3 .

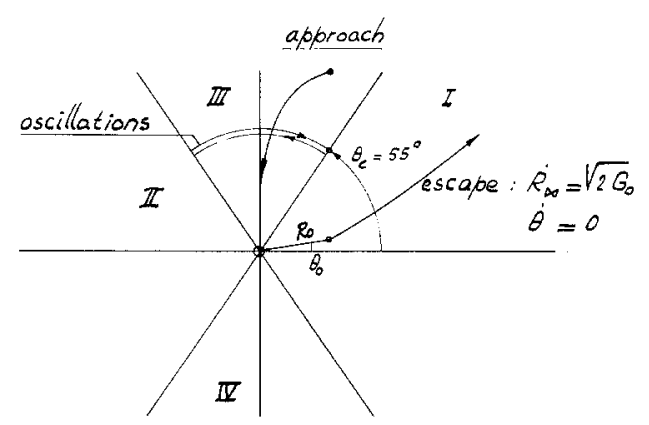

Figure 3. Summary of results for $\mathbf{R}(t)$ according to approximate theory. 
(i) When at $t=0$ the separation vector $\mathbf{R}_{0}$ ends in regions I and II, where $G>0$, the bubbles escape from each other. For $R \gg R_{0}$, $\dot{R} \sim\left(2 G_{0}\right)^{1 / 2}$ and $\dot{\theta} \rightarrow 0$. The angle at which $G$ changes sign is $\theta=\theta_{c} \sim 55^{\circ}$.

(ii) When initially $G<0$, which places $\mathbf{R}_{0}$ in regions III or IV, the bubbles approach each other. The line of centres tends to the vertical in Figure 3 .

(iii) When initially $\theta \approx \theta_{c}$, oscillatory motions are possible. $R$ is approximately constant, which gives, from (21) and (23)

$$
\ddot{\theta}-\Omega^{2} \sin 2 \theta=0, \quad \Omega^{2}=\frac{36 a^{3} U_{0}^{2}}{R_{0}^{5}}
$$

or

$$
\dot{\theta}^{2}=\Omega^{2}\left(\cos 2 \theta_{0}-\cos 2 \theta\right) .
$$

However $R$ is only approximately constant. Eventually the separation distance becomes either large or small. These qualitative properties of trajectories agree quite well with the computed trajectories.

Finally we consider the question of the probability distribution. The pair probability distribution $P(\mathbf{x}, \mathbf{x}+\mathbf{R})$ changes, because of the relative motion, according to

$$
\frac{\partial P}{\partial t}+\nabla \cdot(\mathrm{V} P)=0
$$

It can be shown, see [3], that $\nabla \cdot V=0$, whence

$$
\frac{\mathrm{d} P}{\mathrm{~d} t}=0
$$

This means that if we move along a trajectory in $\mathbf{R}, t$ space, $P$ remains constant. If therefore $P$ is random at $t=0$, e.g. $P=n$, the probability density remains uniform. This is in contrast to what happens in suspensions dominated by viscosity where the probability density is affected by the relative motion, and in some cases cannot even be determined owing to the occurrence of closed trajectories.

\section{References}

1. Batchelor GK (1970) The stress system in a suspension of force-free particles. J Fluid Mech 41: 545.

2. Batchelor GK (1974) Transport properties of two-phase materials with random structure. Ann Rev Fluid Mech 6: 227.

3. Biesheuvel A and Van Wijngaarden $L$ (to be published).

4. Jeffrey DJ (1973) Conduction through a random suspension of spheres. Proc $R$ Soc London A 335: 355.

5. Knibbe P (1981) Master's Thesis. Technological University Twente, The Netherlands. 
6. Landweber L and Miloh T (1980) Unsteady Lagally theorem for multipoles and deformable bodies. J Fluid Mech 96: 33.

7. Voinov OV and Petrov $A G$ (1977). On the stress tensor in a fluid containing disperse particles. PMM 41: 362.

8. Van Beek P (1981) An O( $\alpha$ )-accurate model for liquid-bubble dispersions. Appl Sc Res.

9. Van Wijngaarden $L$ (1976) Hydrodynamic interaction between gas bubbles in liquid. J Fluid Mech 77: 27.

10. Van Wijngaarden L Jn (1980) On the mathematical modeling of two-phase flows. Proc IVth Int Meeting on water column separation, Cagliari, 1979. 